\title{
Clinical experience on diagnosis and treatment for malignancy originating from the dacryocyst
}

\author{
Li-juan Zhu ${ }^{1} \cdot$ Yu Zhu ${ }^{1} \cdot$ Shang-chen Hao ${ }^{1} \cdot$ Pei Huang ${ }^{1} \cdot$ Lin-lin Wang $^{1} \cdot$ Xiu-hong Li ${ }^{1} \cdot$ Li-hong Lian $^{1} \cdot \mathrm{Ke} \mathrm{Zhang}^{1}$
}

Received: 9 February 2018 / Revised: 21 March 2018 / Accepted: 3 May 2018 / Published online: 30 May 2018

(c) The Royal College of Ophthalmologists 2018

\begin{abstract}
Objective To summarize clinical experience on the diagnosis and treatment for malignancy originating from the dacryocyst. Methods We retrospectively analyzed clinicopathological data from 12 cases that were diagnosed with malignancy originating from the dacryocyst by histopathological examination in our hospital from 2007 to 2017.

Results Of the 12 cases with malignancy originating from the dacryocyst, 7 were male and 5 were female, with a mean age of 53 years (range, 4-81). Clinical manifestations included a mass in the dacryocyst area in 12 cases, epiphora in 9 cases, pyoid tears in 2 cases, bloody tears in 3 cases, and redness and swelling in the dacryocyst area in 2 cases. Lymphoma occurred in six cases, malignant melanoma in three cases, embryonal rhabdomyosarcoma in one case, and squamous cell carcinoma in two cases.

Conclusions Misdiagnosis and missed diagnosis readily occur for malignancy originating from the dacryocyst because its clinical manifestations are diverse. For the suspected patients, it is necessary to perform related examinations. Individualized treatment should be adopted based on pathological types and specific conditions.
\end{abstract}

\section{Introduction}

The malignancy originating from the dacryocyst is rare and has various clinical manifestations. Most of malignancies from the dacryocyst exhibit epiphora, which is easily diagnosed as chronic dacryocystitis, delaying diagnosis and treatment. Pathological types of malignancy from the dacryocyst include squamous cell carcinoma, adenocarcinoma, lymphoma, malignant melanoma, rhabdomyosarcoma, and so on. The prognosis of dacryocyst malignancy is related to pathological type and staging, especially early diagnosis and early treatment. At present, there is no unified standard for the treatment of dacryocyst malignancy. Except surgical resection, the therapeutic effects of radiotherapy and chemotherapy on dacryocyst malignancy have been controversial [1-5]. The aim of this study was to further understand the clinical features, pathological types, treatment, and prognosis of dacryocyst

Yu Zhu

64042316@qq.com

1 Department of Ophthalmology, The First Affiliated Hospital of Zhengzhou University, 450052 Zhengzhou, China malignancy by analysis of clinicopathological data from 12 cases.

\section{Materials and methods}

All study methods were approved by the Ethics Committee of the First Affiliated Hospital of Zhengzhou University. All the subjects enrolled into the study gave written informed consent to participate.

We retrospectively analyzed clinical symptoms, radiographic outcomes, histopathological sections, immunohistochemical staining, treatment, and prognosis in the 12 cases that were diagnosed with malignancy originating from the dacryocyst by histopathological examination in our hospital from 2007 to 2017.

\section{Results}

\section{Clinical features}

Of the 12 dacryocyst malignancy cases with a mean age of 53 years (range, 4-81), 7 with a mean age of 46 years were male and 5 with a mean age of 63 years were female. The 
Table 1 Data of 12 cases with malignancy of the lacrimal sac

\begin{tabular}{|c|c|c|c|c|c|c|c|}
\hline Cases & Sex & Age & History & Side & Histology & Treatment & Follow-up (months) \\
\hline 1 & M & 62 & 6 months & Left & MALT & Surgery+RT & No recurrence during 108 months \\
\hline 2 & M & 60 & 4 months & Right & MALT & Surgery+RT & No recurrence during 60 months \\
\hline 3 & $\mathrm{~F}$ & 81 & 2 months & Left & DLBCL & Surgery & Death in 12 months \\
\hline 4 & M & 62 & 10 months & Right & DLBCL & Surgery $+\mathrm{RT}+\mathrm{CT}$ & Death in 36 months \\
\hline 5 & M & 46 & 1 month & Left & $\mathrm{NK} / \mathrm{T}$ & Surgery $+\mathrm{RT}+\mathrm{CT}$ & No recurrence during 60 months \\
\hline 6 & M & 45 & 20 days & Right & $\mathrm{NK} / \mathrm{T}$ & Surgery $+\mathrm{RT}+\mathrm{CT}$ & No recurrence during 6 months \\
\hline 7 & $\mathrm{~F}$ & 63 & 3 years & Left & Malignant melanoma & Surgery & Death in 6 months \\
\hline 8 & $\mathrm{~F}$ & 60 & 2 months & Left & Malignant melanoma & Surgery+RT & No recurrence during 60 months \\
\hline 9 & $\mathrm{~F}$ & 49 & 1 month & Left & Malignant melanoma & Surgery+RT & Death in 60 months \\
\hline 10 & M & 45 & 8 months & Right & Squamous cell carcinoma & Surgery+RT & Recurrence in 60 months \\
\hline 11 & $\mathrm{~F}$ & 61 & 3 months & Right & Squamous cell carcinoma & Surgery+RT & No recurrence during 60 months \\
\hline 12 & M & 4 & 20 days & Left & Rhabdomyosarcoma & Surgery $+\mathrm{RT}+\mathrm{CT}$ & No recurrence during 24 months \\
\hline
\end{tabular}

$F$ female, $M$ male, $R T$ radiotherapy, $C T$ chemotherapy, $D L B C L$ diffuse large B cell lymphoma, MALT extranodal marginal zone B cell lymphoma of mucosa-associated lymphoid tissue, $N K / T$ extranodal NK/T cell lymphoma

dacryocyst malignancy occurred in the left eye of seven cases, and in the right eye of five cases. The shortest course of disease was 20 days and the longest was 3 years. All the 12 cases had a mass in the dacryocyst area. Epiphora occurred in nine cases. Of the nine cases, two also had pyoid tears and three also had malignant melanoma-caused bloody tears. One case with malignant melanoma had epistaxis and exophthalmos with outward displacement. Two cases with NK/T cell lymphoma had redness and swelling in the dacryocyst area, and were misdiagnosed as acute dacryocystitis, which did not respond to antibiotic treatment.

\section{Radiographic outcomes}

CT showed a mass in the dacryocyst area in all the 12 cases, nasolacrimal duct expansion in four cases, including two cases with lymphoma, one case with squamous cell carcinoma, and one case with malignant melanoma, bone destruction involving paranasal sinus and orbit in one case with malignant melanoma, and soft tissue swelling around the orbit in two cases with NK/T cell lymphoma.

\section{Pathological characteristics}

Of the 12 cases, 6 had lymphoma, including extranodal marginal zone B cell lymphoma of mucosa-associated lymphoid tissue in 2 cases, diffuse large B cell lymphoma in 2 cases, and extranodal NK/T cell lymphoma in 2 cases. Three cases had malignant melanoma, one case embryonal rhabdomyosarcoma, and two cases squamous cell carcinoma.

\section{Treatment and prognosis}

In the six cases with lymphoma, two with extranodal marginal zone B cell lymphoma of mucosa-associated lymphoid tissue received surgery followed by postoperative radiotherapy, and follow-up indicated no recurrence. Of the two cases with diffuse large B cell lymphoma, an 81-yearold female case refused chemoradiation after surgery, and died 1 year later; another 62-year-old male case received surgery and postoperative chemoradiation, and died 3 years later. Two cases with NK/T cell lymphoma received surgery and postoperative chemoradiation, and had no recurrence. In three cases with malignant melanoma, a 63-year-old female case with melanoma involving paranasal sinuses and orbit refused chemoradiation after surgery and died 6 months later; other two cases with malignant melanoma received surgery and postoperative radiotherapy, and in the two cases, one 60-year-old female case died 5 years later and another 49-year-old female case still survived. One 4year-old child with embryonal rhabdomyosarcoma received surgery and postoperative chemoradiation and 2-year follow-up indicated no recurrence. Two cases with squamous cell carcinoma received surgery and postoperative radiotherapy; and in the two cases, one 61-year-old female case had no recurrence during 5-year follow-up and another 45year-old male case had oral squamous cell carcinoma during the 5-year follow-up (Table 1).

\section{Discussion}

The malignancy of the lacrimal sac is rare and commonly occurs in 50-60-year-old people. Its incident is not different in sex and races. The malignancy of the lacrimal sac is characterized by insidious onset and lack of specific clinical manifestation, so misdiagnosis and missed diagnosis easily occur and the duration from onset to medical consultation is usually long. In this study, seven cases were male and five were female without significant difference between sex, and 
with a mean age of 53 years (range, 4-81), which is consistent with the results in other reports [1-5].

The malignancy originating from lacrimal sac shows various clinical manifestations such as epiphora and pyorrhea in early stage; local mass in the dacryocyst area, invasion to nasal cavity and orbit, and local skin rupture due to tumor growth; and regional lymphadenopathy and systemic metastasis in advanced stage. In malignancy originating from dacryocyst, the most common clinical symptoms were swelling of lacrimal sac region and epiphora, which are readily diagnosed as chronic dacryocystitis because these symptoms are similar to the clinical symptoms of chronic dacryocystitis. The rare symptoms including bloody tears as well as redness and swelling in the dacryocyst area should be paid attention. In this study, two cases had redness and swelling in the dacryocyst area, which was similar to the symptoms of acute dacryocystitis, did not respond to antibiotics, and were finally diagnosed with extranodal $\mathrm{NK} / \mathrm{T}$ cell lymphoma by pathological examination. The pathology of NK/T lymphoma is characterized by pleomorphic lymphocytic infiltration around blood vessels, tumor cell infiltration leads to necrosis and inflammation of local blood vessel. This easily misdiagnosed as inflammatory lesion. It is difficult to make a definite diagnosis when the sample site is too shallow or improper in pathological biopsy, so multiple pathological biopsies are required if necessary [6]. It has been reported that lymphoma originating from the dacryocyst commonly diffuse large B cell lymphoma (DLBCL) and extranodal marginal zone B cell lymphoma of mucosa-associated lymphoid tissue (MALT), while extranodal NK/T cell lymphoma (NK/T) is rare [7-9]. However, in this study, of the six cases with lymphoma, two had NK/T. Therefore, $\mathrm{NK} / \mathrm{T}$ should not be neglected in the malignancy originating from dacryocyst, and the possibility of NK/T lymphoma should be considered in the cases that have dacryocystitissimilar symptoms and do not respond to antibiotics. Bloody tears may be related to rich blood supply of tumor and tumor growth into the dacryocyst cavity through dacryocyst epithelium [10-13]. In this study, three cases with malignant melanoma all had bloody tears, so the case with bloody tears may have malignancy originating from dacryocysts, especially malignant melanoma.

In the patients with suspected dacryocyst malignancy, it is necessary to know their medical history, examine their eyes, and perform lacrimal passage irrigation and exploration. Nasal endoscopy can find whether there is a mass extending to the lower nasal cavity through the nasolacrimal duct. If a mass is found, biopsy is necessary. In body examination, we should pay attention to lymphadenopathy, especially the lymph node in head and neck. MRI can discriminate between cystic and solid lesions. MRI shows solid lesions as moderate signal intensity in T1-weighted sequence and low-signal intensity in T2-weighted image, while shows cystic lesions caused by dacryocystitis as highsignal intensity in T2-weighted image. Axial and coronal CT scans could determine the extent of lesions, relationship with surrounding tissues, and bone destruction. The malignancy originating from dacryocyst may grow into the nasolacrimal duct, leading to nasolacrimal duct expansion $[14,15]$. If imaging examination shows nasolacrimal duct expansion, especially accompanied by bone destruction; a great deal of attention should be paid to the possibility of malignancy from dacryocyst. In this study, one case with malignant melanoma had bone destruction involving paranasal sinus and orbit, and died 6 months after surgery.

The main treatment methods for malignancy originating from dacryocyst include surgery, radiotherapy, and chemotherapy. For the malignant tumor confined to lacrimal sac, besides the mass, nasolacrimal duct and vicinal periosteum also should be removed during surgery. For most tumors with high malignancy, the resection scope should extend to nasal and orbital bones. For most malignant tumors invading the surrounding tissues, the mass is removed as thoroughly as possible in order to get the margins free from tumor cells. Postoperative radiotherapy can improve the local control rate and reduce tumor recurrence and metastasis rates $[5,7]$. This study indicated that postoperative radiotherapy had a certain effect on the malignancy originating from dacryocyst, especially for MALT because two cases with MALT had no recurrence 108 and 60 months after surgery, respectively. The choice of chemotherapy is based on pathological types and stages. In recent years, the development of immunotherapy and biological target therapy has provided new options for the treatment of malignancy originating from dacryocyst.

Malignancy originating from dacryocyst is rare and there are no unified treatment protocols for it. This study indicated that the prognosis of malignancy originating from dacryocyst is closely associated with pathological types. Therefore, individualized treatment should be adopted based on pathological types, tumor stage, and specific conditions. It has been reported that MALT lymphoma is a low-grade malignant with a good prognosis, while DLBCL and NK/T cell lymphoma are high-grade malignant with a poor prognosis [6-9, 16, 17]. In this study, two cases with MALT had no recurrence 5 years after treatment, but two cases with DLBCL died within 5 years after treatment. Our results were consistent with the reports [6-9, 16, 17]. Therefore, we suggest that for MALT lymphoma confined to lacrimal sac, surgical resection combined with radiotherapy is adopted; for DLBCL and NK/T cell lymphoma, and advanced MALT lymphoma, surgical resection combined with chemoradiation is adopted. Dacryocyst melanoma, mucosa malignant melanoma, is characterized by strong invasion, easily recurrence and metastasis, and poor prognosis [10-13]. This study also 
indicated poor prognosis of malignant melanoma because two cases died within 5 years after treatment in the three cases with malignant melanoma. Therefore, we suggest that for malignant melanoma, extended surgical resection combined with radiotherapy and immunotherapy is adopted. Comprehensive treatment can greatly improve the survival rate of cases with rhabdomyosarcoma although rhabdomyosarcoma is characterized by easily frequent recurrences and metastasis [18]. In this study, one case with rhabdomyosarcoma who was treated by surgical resection combined with chemoradiation, had no recurrence during 2-year follow-up, so we suggest that for rhabdomyosarcoma, surgical resection combined with chemoradiation is adopted. Squamous cell carcinoma in head and neck is characterized by local recurrence and lymphatic metastasis and is sensitive to radiotherapy [19]. In this study, one case with squamous cell carcinoma received surgical resection combined with radiotherapy; although he had no recurrence in his right eye, squamous cell carcinoma occurred in his oral cavity 5 years later. Therefore, we suggest that for squamous cell carcinoma confined to lacrimal sac, surgical resection combined with radiotherapy is adopted; for advanced squamous cell carcinoma, surgical resection combined with chemoradiation is adopted. Due to rare cases with malignancy originating from dacryocyst in clinics, it is necessary to further collect cases to summarize the experience of diagnosis and treatment.

\section{Summary}

\section{What was known before:}

- The malignancy originating from the dacryocyst is rare.

- Most of malignancy from the dacryocyst are easily diagnosed as chronic dacryocystitis.

\section{What this study adds:}

- NK/T should not be neglected in the malignancy originating from dacryocyst.

- The case with bloody tears may have malignancy originating from dacryocysts, especially malignant melanoma.

\section{Compliance with ethical standards}

Conflict of interest The authors declare that they have no conflict of interest.

\section{References}

1. Krishna Y, Coupland SE. Lacrimal sac tumors--a review. J Asia Pac Ophthalmol. 2017;6:173-8.
2. Heindl LM, Jünemann AG, Kruse FE, Holbach LM. Tumors of the lacrimal drainage system. Orbit. 2010;29:298-306.

3. Montalban A, Liétin B, Louvrier C, Russier M, Kemeny JL, Mom T, et al. Malignant lacrimal sac tumors. Eur Ann Otorhinolaryngol Head Neck Dis. 2010;127:165-72.

4. Stephanyszyn MA, Hidayat AA, Pe'er JJ, Flanagan JC. Lacrimal sac tumors. Ophthal Plast Reconstr Surg. 1994;10:169-84.

5. Ni C, D'Amico DJ, Fan CQ, Kuo PK. tumors of the lacrimal sac: a clinicopathologicalanalysis of 82 cases. Int Opthalmol Clin. 1982;22:121-40.

6. Pine RR, Clark JD, Sokol JA. CD56negative extranodal NK/Tcell lymphoma of the orbit mimicking orbital cellulitis. Orbit. 2013;32:45-8.

7. Graue GF, Finger PT, Maher E, Della Rocca D, Della Rocca R, Lelli GJ Jr, et al. Ocular adnexal lymphoma staging and treatment: American Joint Committee on Cancer versus Ann Arbor. Eur J Ophthalmol. 2013;23:344-55.

8. Sjö LD, Ralfkiaer E, Juhl BR, Prause JU, et al. Primary lymphoma of the lacrimal sac: an EORTC ophthalmic oncology task force study. Br J Ophthalmol. 2006;90:1004-9.

9. McKelvie PA, McNab A, Francis IC, Fox R, O’Day J. Ocular adnexal lymphoproliferative disease: a series of 73 cases. Clin Exp Ophthalmol. 2001;29:387-93.

10. Lee HM, Kang HJ, Choi G, Chae SW, Kim CH, Hwang SJ, et al. Two cases of primary malignant melanoma of the lacrimal sac. Head Neck. 2001;23:809-13.

11. Sendra Tello J, Galindo Campillo N, Rodriguez-Peralto JL, Alvarez-Linera J, Garabito Cocina I. Malignant melanoma of the lacrimal sac. Otolaryngol Head Neck Surg. 2004;131:334-6.

12. Li YJ, Zhu SJ, Yan H, Han J, Wang D, Xu S. Primary malignant melanoma of the lacrimal sac. BMJ Case Rep. 2012;2012: bcr2012006349.

13. Kavoussi SC, Levin F, Servat JJ. Orbital extension of untreated lacrimal sac melanoma following dacryocystorhinostomy. Ophthal Plast Reconstr Surg. 2016;32:e76.

14. Rahangdale SR, Castillo M, Shockley W. MR in squamous cell carcinoma of the lacrimal sac. AJNR Am J Neuroradiol. 1995;6:1262-4.

15. Kumar VA, Esmaeli B, Ahmed S, Gogia B, Debnam JM, Ginsberg LE. Imaging features of malignant lacrimal sac and nasolacrimal duct tumors. AJNR Am J Neuroradiol. 2016; Epud ahead of print

16. Ponzoni M, Govi S, Licata G, Mappa S, Giordano Resti A, Politi LS, et al. A reappraisal of the diagnostic and therapeutic management of uncommon histologies of primary ocular adnexal lymphoma. Oncologist. 2013;18:876-84.

17. Desai A, Joag MG, Lekakis L, Chapman JR, Vega F, Tibshirani $\mathrm{R}$, et al. Long-term course of patients with primary ocular adnexal MALT lymphoma: a large single-institution cohort study. Blood. 2017;129:324-32.

18. Raney RB, Walterhouse DO, Meza JL, Andrassy RJ, Breneman JC, Crist WM, et al. Results of the Intergroup Rhabdomyosarcoma Study Group D9602 protocol, using vincristine and dactinomycin with or without cyclophosphamide and radiation therapy, for newly diagnosedpatients with low-risk embryonal rhabdomyosarcoma: a report from the Soft Tissue Sarcoma Committee of the Children's Oncology Group. J Clin Oncol. 2011;29:1312-8.

19. Grégoire V, Lefebvre JL, Licitra L, Felip E, EHNS-ESMOESTRO Guidelines Working Group. Squamous cell carcinoma of the head and neck: EHNS-ESMO-ESTRO Clinical Practice Guidelines for diagnosis, treatment and follow-up. Ann Oncol. 2010;21(Suppl 5):v184-6. 